Research Article

\title{
Design and Performance Evaluation of Porous Titanium Alloy Structures for Bone Implantation
}

\author{
Jianping Shi $\mathbb{D},{ }^{1}$ Huixin Liang, ${ }^{2}$ Jie Jiang, ${ }^{3}$ Wenlai Tang $\mathbb{D},{ }^{1}$ and Jiquan Yang $\mathbb{D}^{1}$ \\ ${ }^{1}$ Jiangsu Key Laboratory of 3D Printing Equipment and Manufacturing, Nanjing Normal University, Nanjing 210042, China \\ ${ }^{2}$ School of Mechanical Engineering, Nanjing University of Aeronautics and Astronautics, Nanjing 210016, China \\ ${ }^{3}$ Jiangsu Key Laboratory of 3D Printing Equipment and Application Technology, Nantong Institute of Technology, \\ Nantong 226002, China \\ Correspondence should be addressed to Wenlai Tang; wltang@njnu.edu.cn and Jiquan Yang; 63047@njnu.edu.cn
}

Received 4 April 2019; Revised 11 July 2019; Accepted 23 August 2019; Published 9 October 2019

Academic Editor: Akhil Garg

Copyright (C) 2019 Jianping Shi et al. This is an open access article distributed under the Creative Commons Attribution License, which permits unrestricted use, distribution, and reproduction in any medium, provided the original work is properly cited.

\begin{abstract}
Implant parts prepared by traditional design and manufacturing methods generally have problems of high stiffness and heavy selfweight, which may cause stress shielding effect between the implanted part and the host bone, and eventually cause loosening of the implanted part. Based on the implicit surface function equations, several porous implant models with controlled pore structure were designed. By adjusting the parameters, the apparent elastic modulus of the porous implant model can be regulated. The biomechanical simulation experiment was performed using CAE software to simulate the stress and elastic modulus of the designed models. The experimental results show that the apparent elastic modulus of the porous structure scaffold is close to that of the bone tissue, which can effectively reduce the stress shielding effect. In addition, the osseointegration status between the implant and the host bone was analyzed by implant experiment. The pushout test results show that the designed porous structures have a good osseointegration effect.
\end{abstract}

\section{Introduction}

At present, design methods for implant part are mostly based on CT (computed tomography) scan, reverse reconstruction, or forward modeling in design software. Implant parts prepared by traditional design and manufacturing methods generally have problems of high stiffness and heavy selfweight, and stress shielding effect would occur between the parts and the host bone after implantation, which may cause loosening of the implant and lead to reducing implant life directly [1-6].

Due to its excellent mechanical properties, good biocompatibility, and low fitting error, porous implants have become hot topics in recent years $[7,8]$. Porous implant parts with a unique interconnected pore structure can promote the formation of mineralized layers on the implant surface and attract protein adsorption. It also facilitates the adhesion, proliferation, and differentiation of osteoblasts and promotes the growth of osteoblasts. At the same time, nutrition and oxygen transport and metabolite excretion can be achieved. In addition, the porous structure is also conducive to the growth of blood vessels and nerves, so it is an ideal part for the implant. However, for porous implant parts, most of the current design methods are the combination of a fully porous structure and a solid model. These methods often face challenges such as low efficiency of the modeling process, low molding quality, or poor biocompatibility. According to the requirements of implant tissue engineering, in addition to the need for biomechanical properties, the porous structure implants must also provide a good microenvironment for the bone tissue cells. The traditional bone implants generally only have the geometric shape of the lesion, and their internal pore structure still needs to be remodeled [9-11].

In the field of artificial implant modeling, the current research mainly focuses on the design of an implant model that combines both mechanical and biological properties. Wang et al. used the Boolean operation of the original solid model and the standard porous model to obtain a bionic scaffold microporous bone model structure [12]. Parthasarathy 
et al. put forward a hierarchical cell structure modeling method for the acetabular cup, making the elastic properties of the implant similar to that of the surrounding bone tissue and achieving biological fixation [13]. Khanoki et al. proposed a heterogeneous lattice structure based on multiscale mechanics and design optimization to reduce the risk of concurrent bone resorption and graft failure [14]. Gibson et al. optimized the design of components for the requirements of the overall mechanical structure of the implanted parts, and the optimized design rules broke many traditional limitations [15]. Porous implant parts constructed by these methods have less consideration for the influence of the porous structure on the implant and the surrounding tissue [16-19].

Porous structural implants are important matrices for the construction of tissue-engineered bone tissue, and they are also important microenvironment providers that bone cells rely on in the growth process. Therefore, it is required that the implant not only needs to have a precise geometric shape of the bone tissue part of the lesion but also needs a certain number of interconnected pores inside the implant. The most important thing is to ensure that the mechanical properties are close to the bone tissue and to avoid stress shielding between them.

Because of the impressive capability of producing porous structures with a controlled architecture precisely owing to their layer-wise building approach and direct link with a computer-aided design (CAD) model, 3D printing (also called additive manufacturing) techniques have been described as crucial production techniques towards a more controlled bone scaffolds [20]. The selective laser melting (SLM) process is one of the recently developed additive manufacturing techniques that emerged in the late $1980 \mathrm{~s}$ and early 1990s [21], which can create functional, complex metal parts directly, bringing a high degree of freedom to design works. Compared to conventional manufacturing techniques, SLM offers a wide range of advantages, such as reduction of production steps, a high level of flexibility, a high material use efficiency, and a near net shape production [22]. Moreover, due to the additive and layer-wise production, the SLM process is capable of producing complex geometrical features that cannot be obtained using conventional production routes. And the SLM-built porous biological metal scaffolds have presented positive results in both in vitro and in vivo studies [23].

Based on the technical characteristics of $3 \mathrm{D}$ printing technology, this article takes the functional design as the guiding ideology to study the modeling methods of porous structural implant parts. The simulation tests and implantation experiments of the designed porous parts were carried out to analyze their mechanical properties and biocompatibility. The results show that the biological properties and mechanical properties of the porous implant parts achieve the desired consequences.

\section{Materials and Methods}

2.1. Model Designing. Through the different adjustments of the combined transformation of triangular periodic functions, a series of implicit surfaces with periodic changes can be constructed. In the constructed surface function, the most representative surface is a three-dimensional periodic miniaturized surface (TPMS). Three-dimensional periodic miniaturized surfaces are approximately defined as follows:

$$
\varnothing(r)=\sum_{k=1}^{K} A_{k} \cos \left[\frac{2 \pi\left(h_{k} \cdot r\right)}{\lambda_{k}}+p_{k}\right]=C,
$$

where $A_{k}$ denotes the amplitude factor, $h_{k}$ denotes the vector of the $k$-th grid in the derivative space, $r$ denotes the position vector of the Euclidean space, $\lambda_{k}$ denotes the periodic wavelength value of the function, $p_{k}$ denotes the function phase offset, and $C$ denotes a constant.

Based on equation (1), in order to obtain richer porous structural units, a function or constant is usually added to the implicit surface function. Taking $P$ surface as an example, a distance constant $t$ is added to its constructor to form a new $P_{t}$ surface, and its construction equation can be shown by the following equation:

$$
f_{p}=a \cos (x)+b \cos (y)+c \cos (z)+t,
$$

where distance constant $t$ is used to express the value of the point distance on the minimal surface from the implicit function $\left(f_{p}(x, t)\right)$. When the distance $t$ is smaller than a certain threshold, the surfaces represented by the implicit function are continuous, and the surface is graphically represented as shown in Figure 1(a). When the distance $t$ is greater than a certain constant threshold, the surface represented by the implicit function will no longer be continuous, and the surface is graphically represented as shown in Figure 1(c). At this time, the implicit function is no longer suitable for modeling of a continuous porous structure. It is usually possible to obtain a porous structure model with different volume fractions by changing the value of $t$.

Three kinds of porous structural models were designed, and the values of parameter $t$ in the implicit function equations are shown in Table 1 . The design specifications of the three cylinders were $\Phi 8 \mathrm{~m} \times 10 \mathrm{~mm}$, and the models are shown in Figure 2.

2.2. FEA Simulation. The finite element simulation (FEA) software ABAQUS was used to simulate and test the porous model. In the simulation process, the porous model was set to a linear, elastic, and homogeneous model. The elastic modulus of the part material was set to $105 \mathrm{GPa}$, Poisson's ratio was 0.31 , and the yield strength was set to $830 \mathrm{MPa}$. The loading values and boundary conditions for the model simulation were described in a previous article published by the author [24]. To facilitate the simulation analysis, a lowthickness cylinder was added to both ends of the porous structure to ensure that each porous structural model exerts the same force. A compressive stress of $30 \mathrm{MPa}$ was applied at the top of the model. The bottom of the model is completely restrained and fixed. The porous model was mesh subdivided. Models 1, 2, and 3 were meshed with 968335, 833442, and 700461 tetrahedral elements, respectively. Through the calculation iteration, the strain and stress distribution of the model was finally obtained. 




(a)

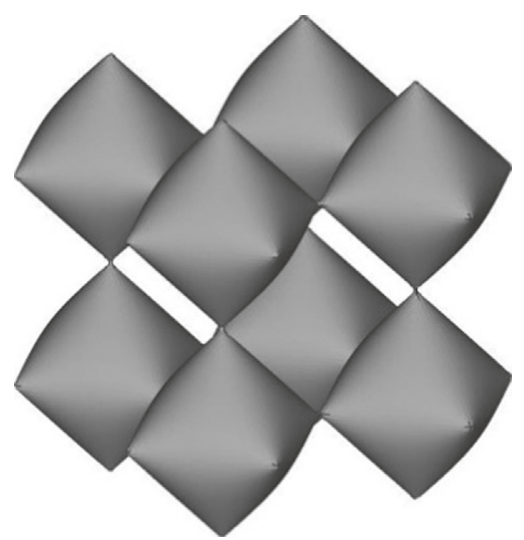

(b)

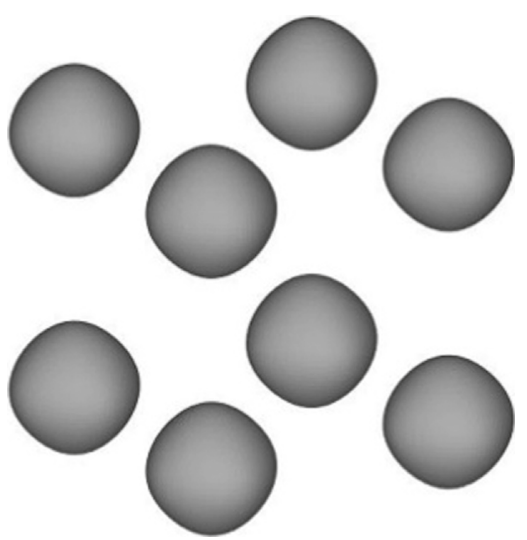

(c)

Figure 1: $P$ surfaces represented by implicit functions corresponding to different distance constants $t$ : (a) 0.5 ; (b) 1 ; (c) 2 .

TABle 1: Porous implant model corresponding to different values of the parameter $t$.

\begin{tabular}{lccc}
\hline Models & 1 & 2 & \\
\hline Values of the parameter $t$ & -0.4 & 0 & 3 \\
Diameter of pore $(\mathrm{mm})$ & 0.37 & 0.50 & 0.4 \\
\hline
\end{tabular}

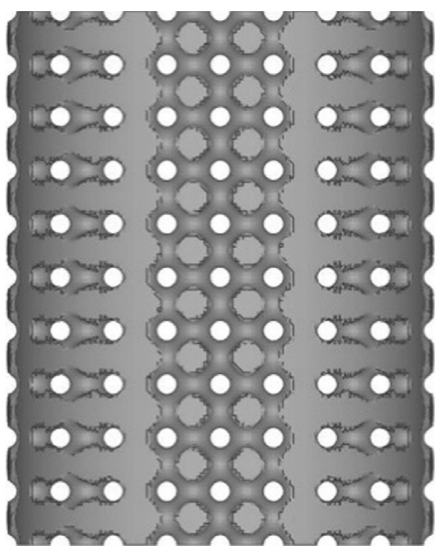

(a)

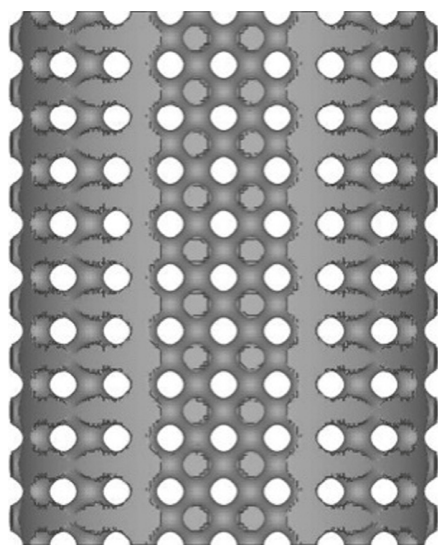

(b)

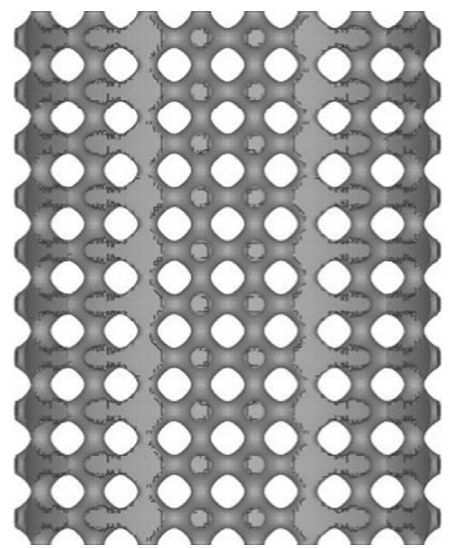

(c)

Figure 2: Three kinds of porous structure models. (a) Model 1. (b) Model 2. (c) Model 3.

2.3. 3D Printing. The Ti6Al4V titanium alloy powder produced by the German EOS company was used as the printing powder material. The composition and mass fractions of the metal powder are shown in Table 2.

The specimens were fabricated with an SLM machine (EOSINT M290; EOS GmbH, Germany) using the optimized processing parameters (Table 3). Selective laser melting of porous scaffolds is characterized by a controllable and precise layer-wise material addition process that allows generating complex structures by selectively melting successive layers of metal powder, using a focused and computer-controlled laser beam, as seen in Figure 3.

2.4. Animal Experiment. A total of 6 male Bama minipigs were enrolled in this study (6 months old). All animal studies
TABLE 2: Chemical composition of titanium alloy powder (\% by mass fraction).

\begin{tabular}{ccccccccc}
\hline Material & $\mathrm{Ti}$ & $\mathrm{Al}$ & $\mathrm{V}$ & $\mathrm{O}$ & $\mathrm{Fe}$ & $\mathrm{C}$ & $\mathrm{N}$ & $\mathrm{H}$ \\
\hline Content & $\mathrm{Bal}$ & 6.00 & 4.00 & 0.15 & 0.10 & 0.03 & 0.01 & 0.01 \\
\hline
\end{tabular}

(including the euthanasia procedure which was performed using lidocaine and propofol) were done in compliance with the regulations and guidelines of the ethics committee of Drum Tower Hospital affiliated to Medical School of Nanjing University and conducted according to the ARRIVE and the IACUC guidelines.

All the implants were implanted at the distal region and proximal region of the right tibia bone. Cefuroxime sodium was intramuscularly injected for 3 days after operation to avoid infection. Tetracycline hydrochloride and Calcein 
TABLE 3: Optimized process parameters used in SLM fabrication.

\begin{tabular}{lccccc}
\hline Laser power & Scan speed & Hatch spacing & Layer thickness & Laser focus & Atmosphere \\
\hline $180 \mathrm{~W}$ & $1200 \mathrm{~mm} / \mathrm{s}$ & $0.1 \mathrm{~mm}$ & $0.03 \mathrm{~mm}$ & $0.08 \mathrm{~mm}$ & Ar \\
\hline
\end{tabular}

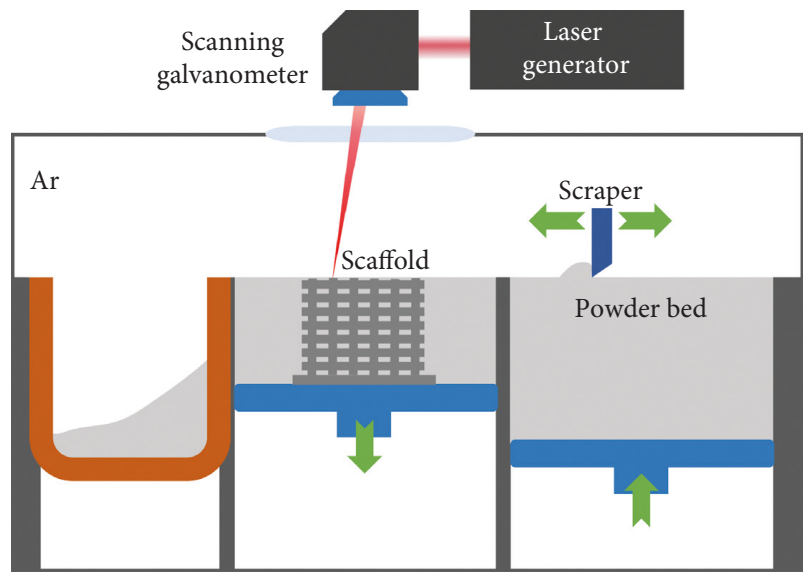

FIGURE 3: Sketch map of SLM printing process.

were injected for the following fluorescence observation. All animals were sacrificed at the 5 th week after operation to measure the osseointegration between the implants and the surrounding bone tissue.

2.5. Performance Evaluation. Micro-CT scan, histological analysis, and biomechanical testing were used to observe the parts of the porous implant. The tibia bones were harvested and scanned in a M1001 micro-CT system (Hiscan Information Technology, Jiangsu, PRC) at $90 \mathrm{kV}, 89 \mu \mathrm{A}$, field of view (FOV) of $63.9 \mathrm{~mm}$, and a nominal isotropic image voxel size of $62.4 \mu \mathrm{m}$. The obtained images were converted to DICOM files for the following analysis.

The harvested femurs were fixed in formalin and then rinsed with distilled water, dehydrated through graded alcohols, and embedded in polymethyl methacrylate (PMMA) without decalcification. Thin sections of $30 \mu \mathrm{m}$ thickness were cut using a diamond-coated saw (310 CP; EXAKT, Germany). The sections were stained with Goldner trichrome staining and then observed using a fluorescence microscope (Olympus Corp., Tokyo, Japan).

In order to test the bonding strength between the porous implant and the host bone, the porous implants were subjected to release experiment test, and the test principle is shown in Figure 4(a). The removed sacrum samples were repaired and fixed on the test platform of the electronic dynamic and static material testing machine (ElectroPuls 3000, Instron, USA). The top punch of the test instrument was vertically aligned with the porous metal implant. During the test, a slow feed was applied at a loading speed of $0.1 \mathrm{~mm} /$ s, as shown in Figure 4(b).

\section{Results}

3.1. Physical Characteristic. The EOS metal 3D printing device was used to print the designed porous structure model, and the printed porous titanium alloy parts are shown in Figure 5. Figure 5(a) is a side view of the printed parts, and Figure 5(b) is a top view of the printed parts. By observing, the parts are structurally complete, without missing or collapse. The surface of the part has fine pores in addition to the designed holes, and the surface roughness of the parts is high.

Figure 6 shows the SEM images of the printed porous parts. It can be seen from the figure that the pore structure of the three structures are different in size. Through measurement, the diameters of the holes in samples 1,2 , and 3 are about $382 \mu \mathrm{m}, 513 \mu \mathrm{m}$, and $618 \mu \mathrm{m}$, respectively. These structural modalities are fully consistent with the model design structure, which infers that the printing accuracy is completely satisfied with the design requirements. In addition, as can be seen from the SEM images, there are fine microstructures on the surface of the part, and the diameters are about $10-100 \mu \mathrm{m}$. According to studies, these types of pore structures are very favorable for the adhesion and proliferation of bone tissue cells.

3.2. FEA Result. Compression performance is a very important mechanical indicator of porous implants. In Figure 7 , the simulation test results show that the designed porous structure exhibits different mechanical properties. As the pore morphology changed, the resulting stress conditions also changed. The compression experiment results show that the stress cloud diagram of each model has its own characteristics by applying the same effect of compressive stress. With the increase of pressure load, the maximum stress values in Model 1, Model 2, and Model 3 gradually increased, which were 188.41 MPa, 285.25 MPa, and 385.30 MPa, respectively.

During the experiment, the total compressive deformation of Model 1 is the smallest of the three models. Due to the smallest structural pore size, the overall compressive stress experienced by the model is approximately evenly divided, and the maximum stress in Model 1 is also the smallest. On the contrary, the total compressive deformation of Model 3 is the largest, the model pore is the largest, and the compressive strength of the model structure is the weakest. Under the same load conditions, Model 3 was first crushed, indicating that the parts with larger pore diameters in the internal pore structure of the model have poor pressure resistance. According to the simulation data, the apparent elastic modulus of Model 1 is the largest, Model 3 is the smallest, and the characteristics of Model 2 are between Model 1 and Model 3. Among them, the apparent elastic modulus of Model 3 is the closest to the elastic modulus of bone tissue.

3.3. Osseointegration Properties. The results of pushout test to verify the osseointegration properties of porous implants 


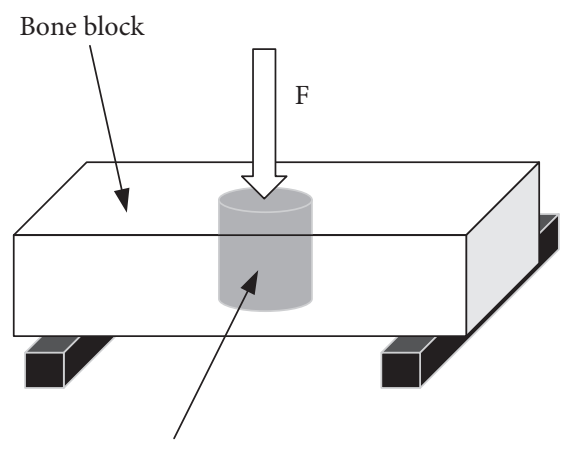

Porous implant

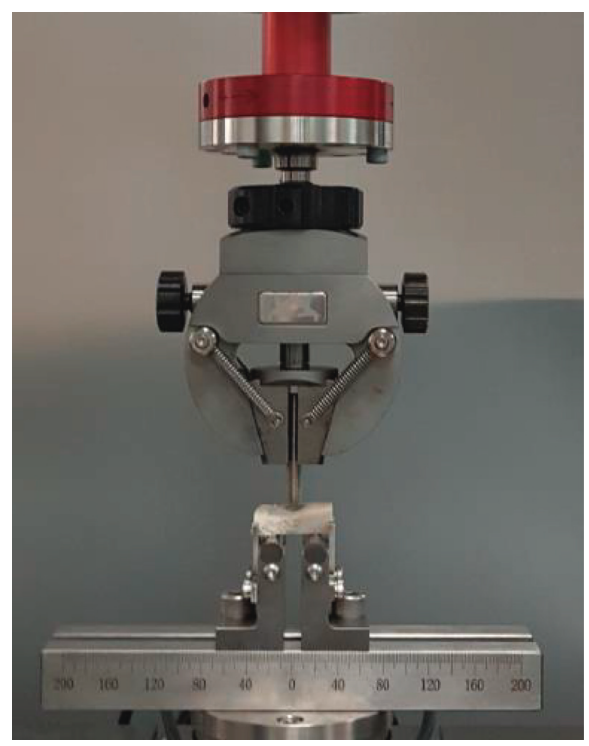

(b)

Figure 4: Pushout test of implant. (a) Schematic diagram of the pushout test and (b) the device of release experiment.

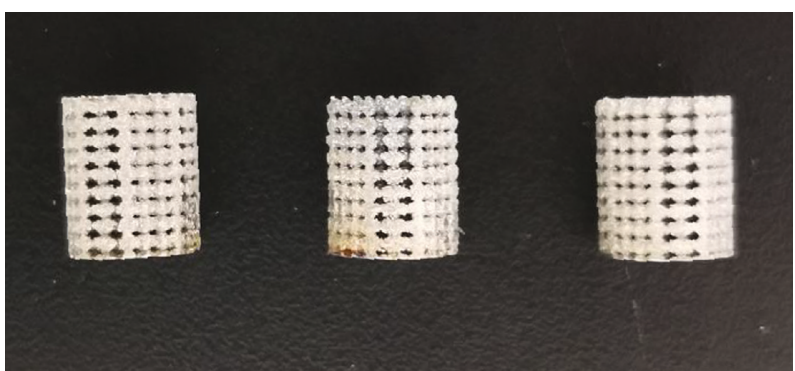

(a)

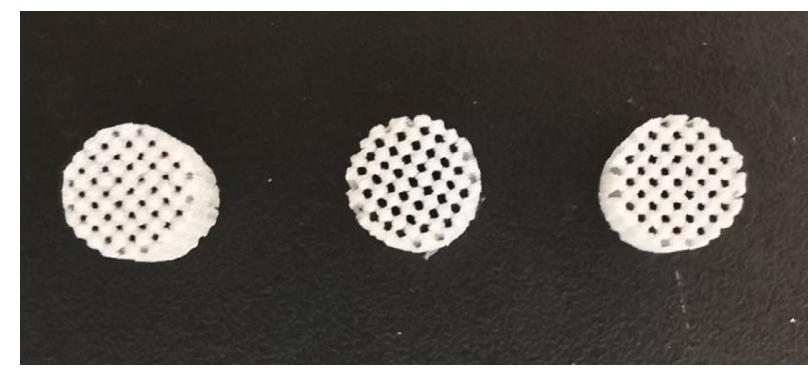

(b)

FIGURE 5: 3D printed titanium porous parts. (a) Side view of the printed parts. (b) Top view of the printed parts.

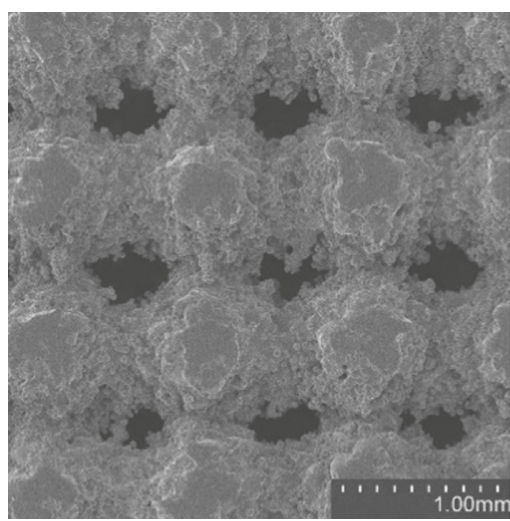

(a)

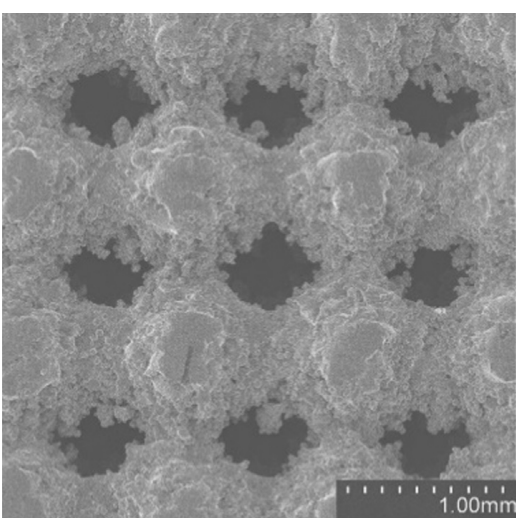

(b)



(c)

Figure 6: SEM images of the printed porous parts. (a) Model 1. (b) Model 2. (c) Model 3.

are shown in Figure 8. It shows the bending stress and strain curves of the samples, and the curve is close to the bending strength curve of bone. All samples have a period of linear elastic strain. When a certain maximum value is reached, the curve turns. At this time, the implant should be slid in the host bone under the action of thrust and finally pushed out with the continuous application of thrust. The stress and strain curves of Sample 1 and Sample 2 are similar. The 


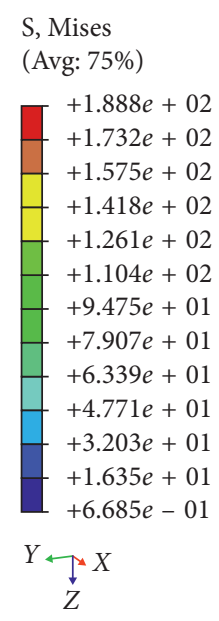

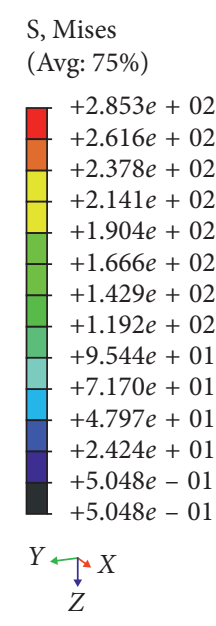

(a)



(b)
S, Mises

(Avg: 75\%)

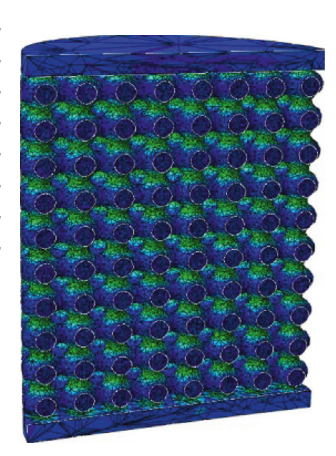

1
$Z$

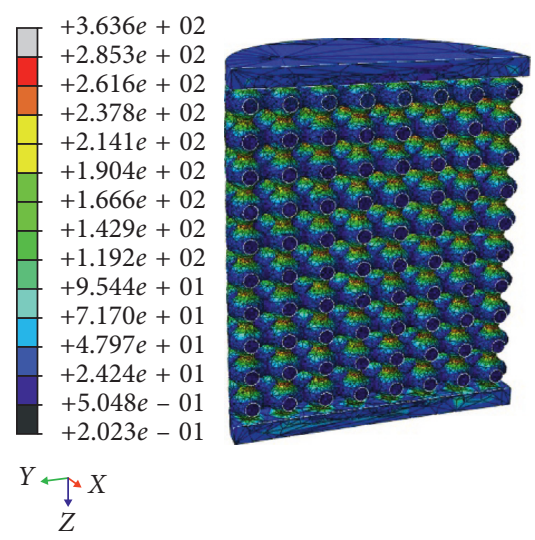

(c)

Figure 7: Stress cloud of porous structure compression simulation. (a) Model 1. (b) Model 2. (c) Model 3.

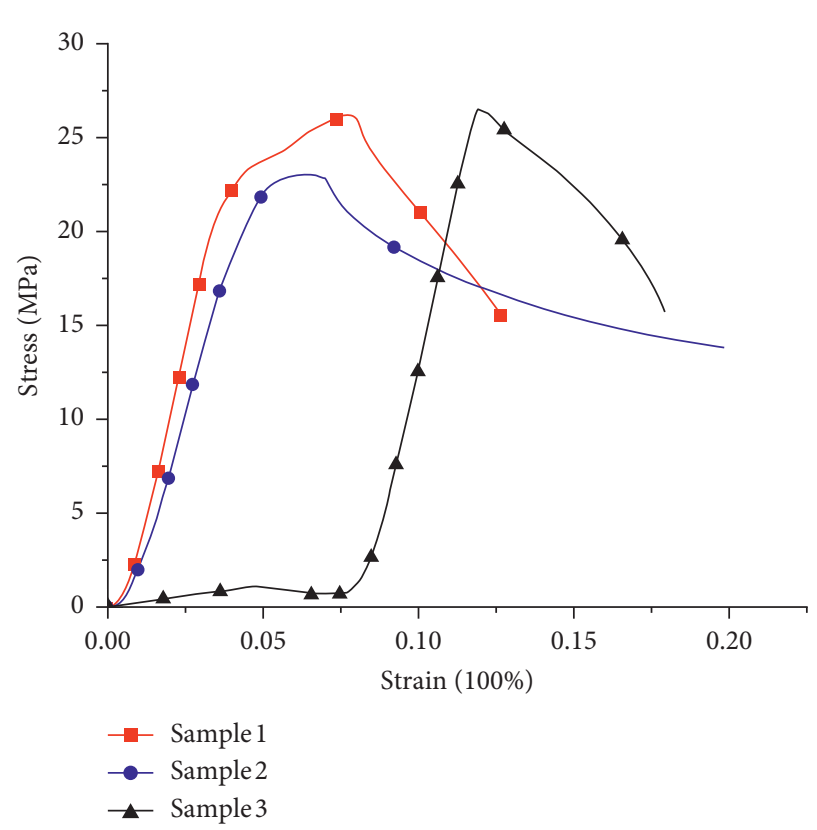

FIgURE 8: Osseointegration properties of the samples.

entire curve of Sample 3 is shifted, which may be caused by the false preloading of Sample 3 when the implant part is clamped on the test equipment. In the figure, the curves of the initial elastic phases of the three structures are basically close. This is due to the fact that the implants of the implant and the host bone are relatively firm at the initial stage. The curve at this time mainly represents the bending characteristics of the bone tissue when compressed. The experimental results showed that the strength of Sample 3 combined with the bone tissue was the best, its ultimate pushout stress reached $27 \mathrm{MPa}$, the implant basically had no slippage during the pushout process, and the whole process had a good linearity. The bond strength between Sample 2 and bone tissue is relatively weak, and its ultimate pushout stress is about $22.5 \mathrm{MPa}$. Sample 1 also had a large ultimate pushing stress, but slip occurred during its ejection.
3.4. Histological Analysis. The growth situation of the bone tissue around the implanted scaffolds is shown in Figure 8. Figure 9(a) shows the micro-CT image of the tibia bones with the implanted porous scaffolds, the areas indicated by the arrow in the figure indicate the formation of the new bone. The results of fluorescent staining of the scaffold bone tissue section are shown in Figure 9(b). It can be seen that the new born osteogenic tissue are tightly wrapped around the porous scaffolds, and the new growth bone can be observed from inside the pores of the scaffolds. Figure 9(c) shows the results of hard tissue section staining in which the black areas represent the scaffold and the blue-green areas represent the new bone tissue. From the above three detection methods, the tight bond between the new bone and the porous scaffolds can be observed, and the bone can also be found to grow inside the scaffolds. This is also the reason for the excellent performance of the launch force in biomechanical testing.

\section{Discussion}

In the present study, we developed several porous implant models with controlled pore structure, and the apparent elastic modulus of the porous implant model can be regulated by adjusting the parameters.

There are many factors that affect the growth of bone tissue into porous implants, and the pore morphology, pore size, and implant biocompatibility are all important factors affecting osseointegration. The porous structure in the implant not only mechanically locks the interface between the implant and the host bone, but it can also promote the proliferation of osteoblasts when the pore size can be reduced to the cellular level $(1-100 \mu \mathrm{m})$. The porous titanium alloy implant components designed and printed in this study have complex pore structures. The experimental results show that three types of porous titanium alloy implant components can be combined with bone tissue after implantation. According to the pushout test, Sample 3 has the best bonding strength among all implanted parts. There may 

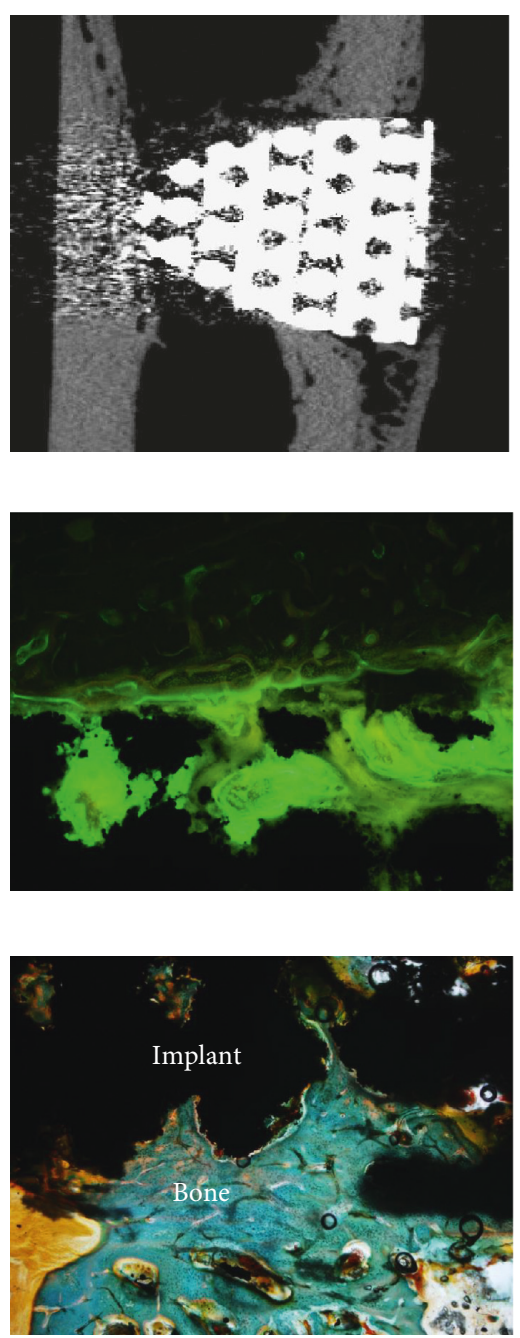


(a)
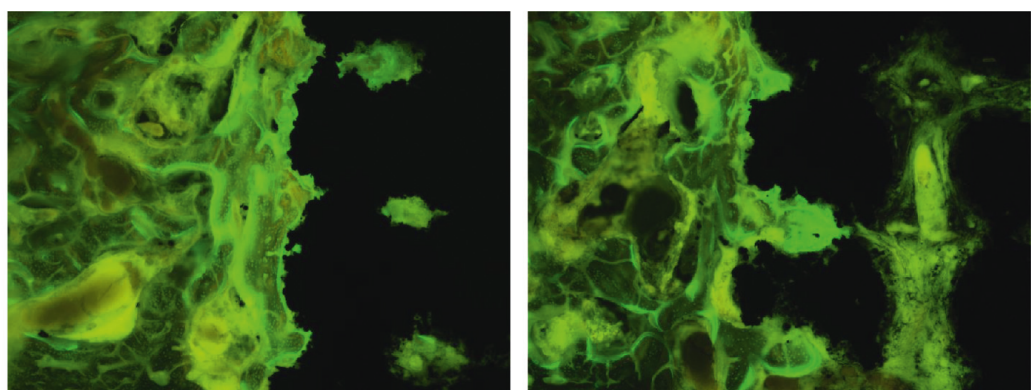

(b)
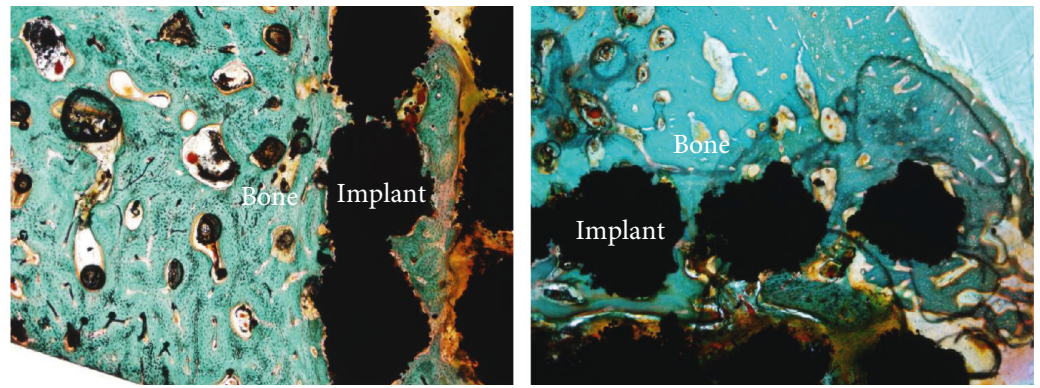

(c)

Figure 9: The growth situation of the bone tissue around the implanted scaffolds. (a) Micro-CT image of the tibia bones with the implanted porous scaffolds; (b) results of fluorescent staining of the scaffold; (c) the results of hard tissue section staining.

be two reasons: on the one hand, the pore size in Sample 3 is suitable for bone tissue growth, and on the other hand, the mechanical properties of Sample 3 are close to those of bone tissue, which can promote bone tissue growth and avoid stress-shielding between bone and implanted parts.

Traditional scaffold fabrication methods such as solvent casting, gas pore forming, and electrospinning often fail to produce satisfactory three-dimensional scaffolds and internal microstructures. 3D printing technology can print out scaffolds with good mechanical properties, adjustable pores, and complex spatial structures. With the advent of $3 \mathrm{D}$ printing technology, scaffolds with porous structures designed by different methods can be manufactured. A traditional porous design method cannot achieve the ideal structure of scaffold design. In this study, the porous scaffold based on TPMS parameterized construction has continuous curvature and controllable pore structure, which can promote the growth of bone tissue [25-27].

At present, one of the main causes of bone graft loosening is that the bond between the graft and the bone is not firm. In this study, an integrated porous bone-promoting graft was made directly through the TPMS design method and $3 \mathrm{D}$ printing technology. It was verified by the mechanical test and animal implantation experiments. The experimental results show that the apparent elastic modulus of the porous structure scaffold is close to that of the bone tissue, which can effectively reduce the stress shielding effect. And the pushout test results show that the designed porous structures have a good osseointegration effect.

\section{Conclusion}

The porous titanium alloy implants studied in this study can improve the interface area between the porous implant and the host bone, and the porous structure of the interconnecting pores in the porous titanium alloy can provide an effective channel for the vascularization of bone tissue, which provides the necessary conditions for the growth of bone tissue. In addition, the mechanical property mismatch between the implant and the host bone is a major cause of 
the resulting stress-shielding effect at the binding interface, which is also responsible for the loosening of implant components after implantation. The porous structure designed in this paper can effectively regulate the apparent elastic modulus of the porous implant. By making the elastic modulus between the implant and the host bone close, the feature facilitates the conduction of stress at the interface, which reduces or eliminates stress shielding between the implant and the host bone. Therefore, a suitable structure of the porous implant is more conducive to the formation of osseointegration of the implant and the bone tissue, which has practical significance for improving the initial and longterm stability of the implant. The method brings new ideas for clinical treatment.

\section{Data Availability}

The data used to support the findings of this study are available from the corresponding author upon request.

\section{Conflicts of Interest}

The authors declare that there are no conflicts of interest regarding the publication of this paper.

\section{Acknowledgments}

This research was funded by Key Technology RD Program of Jiangsu Province under Grants BE2018010 and BE2016010, the National Natural Science Foundation of China (grant nos. 51805272, 61273243, 51705259, and 51607094), the Natural Science Research Projects of Jiangsu Higher Education Institution (19KJD460005), Key Laboratory Fund of Provincial Science and Technology Department (BM2013006), Open fund of Jiangsu Key Laboratory of 3D Printing Equipment and Application Technology (2018KFKT02), and Nantong Key Laboratory of 3D Printing Technology and Application (CP12016002).

\section{References}

[1] W. V. Grunsven, E. Hernandeznava, G. C. Reilly et al., "Fabrication and mechanical characterisation of titanium lattices with graded porosity," Metals, vol. 4, no. 3, pp. 401-409, 2014.

[2] J. Shi, J. Yang, Z. Li, L. Zhu, L. Li, and X. Wang, "Design and fabrication of graduated porous Ti-based alloy implants for biomedical applications," Journal of Alloys and Compounds, vol. 728, pp. 1043-1048, 2017.

[3] J. Brezinová, R. Hudák, A. Guzanová, D. Draganovská, G. Ižaríková, and J. Koncz, "Direct metal laser sintering of Ti6Al4V for biomedical applications: microstructure, corrosion properties, and mechanical treatment of implants," Metals, vol. 6, no. 7, pp. 171-179, 2016.

[4] H. Shen and L. C. Brinson, "A numerical investigation of porous titanium as orthopedic implant material," Mechanics of Materials, vol. 43, no. 8, pp. 420-430, 2011.

[5] L. Yan, Y. Yuan, L. Ouyang, H. Li, A. Mirzasadeghi, and L. Li, "Improved mechanical properties of the new Ti-15Ta- $x \mathrm{Zr}$ alloys fabricated by selective laser melting for biomedical application," Journal of Alloys and Compounds, vol. 688, pp. 156-162, 2016.
[6] J. Shi, J. Yang, L. Zhu, L. Li, Z. Li, and X. Wang, "A porous scaffold design method for bone tissue engineering using triply periodic minimal surfaces," IEEE Access, vol. 6, pp. 1015-1022, 2017.

[7] D. J. Cossetto and A. D. Gouda, "Uncemented tibial fixation total knee arthroplasty," The Journal of Arthroplasty, vol. 26, no. 1, pp. 41-44, 2009.

[8] C. Yan, L. Hao, A. Hussein, and P. Young, "Ti-6Al-4V triply periodic minimal surface structures for bone implants fabricated via selective laser melting," Journal of the Mechanical Behavior of Biomedical Materials, vol. 51, pp. 61-73, 2015.

[9] C. Schroeder, W. C. Regli, A. Shokoufandeh et al., "Computer-aided design of porous artifacts," Computer-Aided Design, vol. 37, no. 3, pp. 339-353, 2005.

[10] A.-V. Do, B. Khorsand, S. M. Geary, and A. K. Salem, "3D printing of scaffolds for tissue regeneration applications," Advanced Healthcare Materials, vol. 4, no. 12, pp. 1742-1762, 2015.

[11] Y. Liu, Q. Lian, J. He, J. Zhao, Z. Jin, and D. Li, "Study on the microstructure of human articular cartilage/bone interface," Journal of Bionic Engineering, vol. 8, no. 3, pp. 251-262, 2011.

[12] H. Wang, S. Johnston, and D. Rosen, "Design of a graded cellular structure for an acetabular hip replacement component," in Proceedings of the 17th Solid Freeform Fabrication, pp. 111-123, Austin, TX, USA, August 2006.

[13] J. Parthasarathy, B. Starly, and S. Raman, "A design for the additive manufacture of functionally graded porous structures with tailored mechanical properties for biomedical applications," Journal of Manufacturing Processes, vol. 13, no. 2, pp. 160-170, 2011.

[14] A. S. Khanoki and D. Pasini, "Multiscale design and multiobjective optimization of orthopedic hip implants with functionally graded cellular material," Journal of Biomechanical Engineering, vol. 134, no. 3, Article ID 031004, 2012.

[15] I. Gibson, D. W. Rosen, and B. Stucker, Additive Manufacturing Technologies: Rapid Prototyping to Direct Digital Manufacturing, ISBN 14419111979781441911193 , Springer US, New York City, NY, USA, 2010.

[16] X. Li, C. Wang, L. Wang, W. Zhang, and Y. Li, "Fabrication of bioactive titanium with controlled porous structure and cell culture in vitro," Rare Metal Materials and Engineering, vol. 39, no. 10, pp. 1697-1701, 2010.

[17] J. Shi, L. Zhu, Z. Li, J. Yang, and X. Wang, "A design and fabrication method for a heterogeneous model of 3D bioprinting," IEEE Access, vol. 5, pp. 5347-5353, 2017.

[18] Y. Huang, X. F. Zhang, G. Gao, T. Yonezawa, and X. Cui, “3D bioprinting and the current applications in tissue engineering," Biotechnology Journal, vol. 12, no. 8, Article ID 1600734, 2017.

[19] A. El-Hajje, E. C. Kolos, J. K. Wang et al., "Physical and mechanical characterisation of 3D-printed porous titanium for biomedical applications," Journal of Materials Science: Materials in Medicine, vol. 25, no. 11, pp. 2471-2480, 2014.

[20] K. F. Leong, C. M. Cheah, and C. K. Chua, "Solid freeform fabrication of three-dimensional scaffolds for engineering replacement tissues and organs," Biomaterials, vol. 24, no. 13, pp. 2363-2378, 2003.

[21] J.-P. Kruth, G. Levy, F. Klocke, and T. H. C. Childs, "Consolidation phenomena in laser and powder-bed based layered manufacturing," CIRP Annals, vol. 56, no. 2, pp. 730-759, 2007.

[22] L. Thijs, F. Verhaeghe, T. Craeghs, J. V. Humbeeck, and J.-P. Kruth, "A study of the microstructural evolution during 
selective laser melting of Ti-6Al-4V," Acta Materialia, vol. 58, no. 9, pp. 3303-3312, 2010.

[23] P. H. Warnke, T. Douglas, P. Wollny et al., "Rapid prototyping: porous titanium alloy scaffolds produced by selective laser melting for bone tissue engineering," Tissue Engineering Part C: Methods, vol. 15, no. 2, pp. 115-124, 2009.

[24] J. Shi, L. Zhu, L. Li et al., "A TPMS-based method for modeling porous scaffolds for bionic bone tissue engineering," Scientific Reports, vol. 8, no. 1, p. 7395, 2018.

[25] F. S. L. Bobbert, K. Lietaert, A. A. Eftekhari et al., "Additively manufactured metallic porous biomaterials based on minimal surfaces: a unique combination of topological, mechanical, and mass transport properties," Acta Biomaterialia, vol. 53, pp. 572-584, 2017.

[26] A. A. Zadpoor, "Bone tissue regeneration: the role of scaffold geometry," Biomaterials Science, vol. 3, no. 2, pp. 231-245, 2015.

[27] G. Li, W. Lei, P. Wei et al., "In vitro and in vivo study of additive manufactured porous Ti6Al4V scaffolds for repairing bone defects," Scientific Reports, vol. 6, no. 1, p. 34072, 2016. 


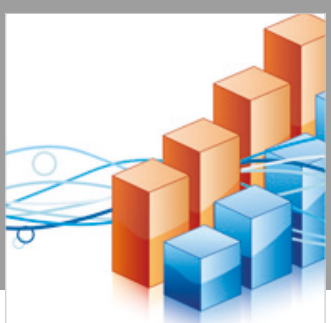

Advances in

Operations Research

\section{-n-m}

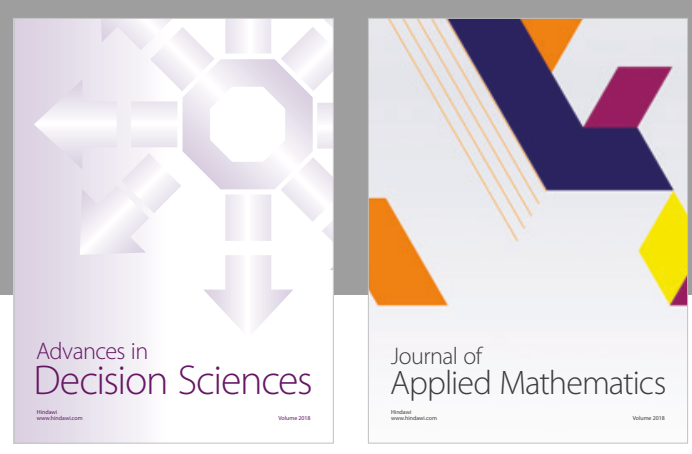

Journal of

Applied Mathematics
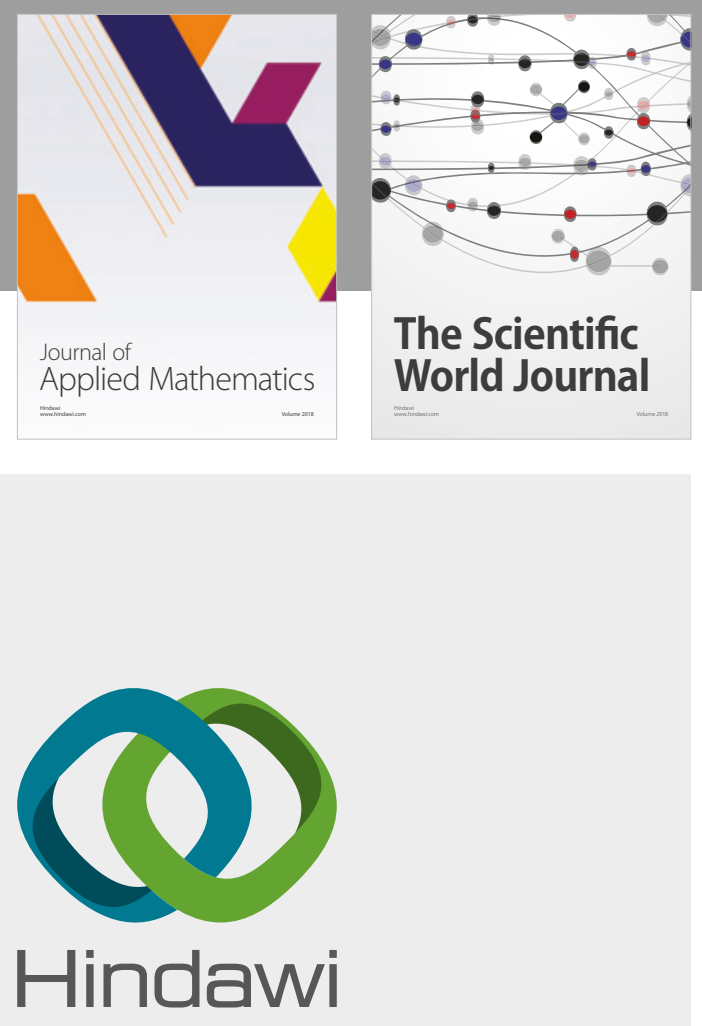

Submit your manuscripts at

www.hindawi.com

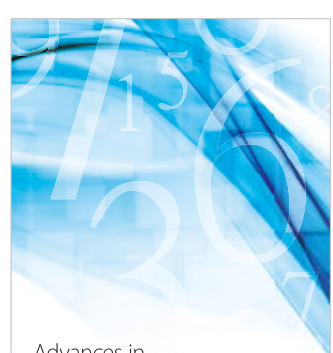

Advances in
Numerical Analysis


Mathematical Problems in Engineering

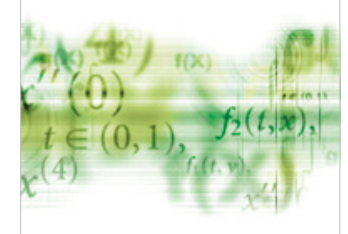

International Journal of

Differential Equations

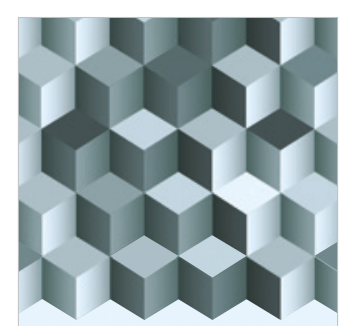

Journal of

Function Spaces

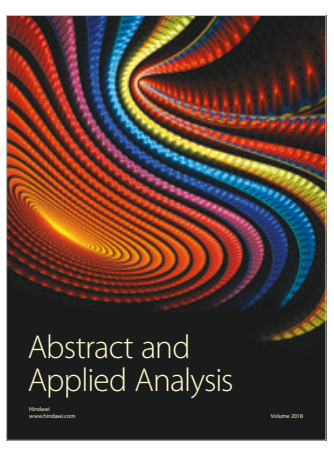

The Scientific

World Journal

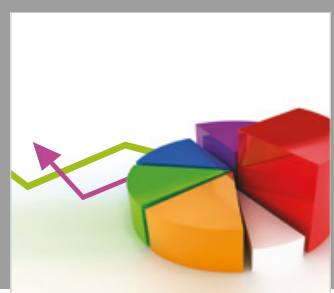

Journal of

Probability and Statistics
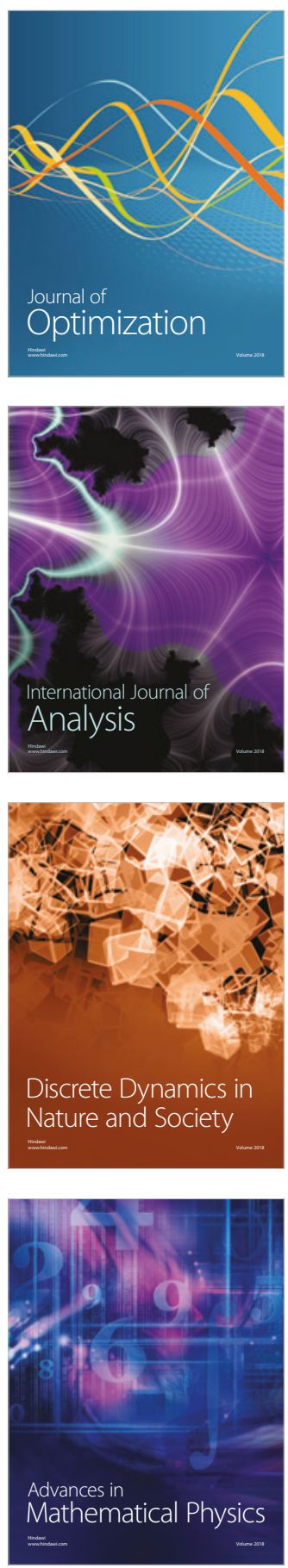